\title{
Predominance of pressure transport in spatial energy budget for a mixing layer approaching absolute instability
}

\author{
A. B. Aadhishwaran* and Sourabh S. Diwan $\odot^{\dagger}$ \\ Department of Aerospace Engineering, Indian Institute of Science, Bengaluru 560012, India
}

(Received 16 March 2021; accepted 25 August 2021; published 13 September 2021)

\begin{abstract}
In this Letter, we report the outcome of a spatial energy budget performed for the linear convective instability of the plane incompressible mixing layer within the inviscid framework. We find that as the critical condition for the onset of absolute instability is approached, the integrated pressure-transport term becomes increasingly more prominent as compared to the integrated production term, and the former dominates the energy budget completely at the critical condition. This implies that, near the threshold of absolute instability, the growth of disturbances is almost entirely due to the pressure-transport mechanism (rather than the more common production mechanism), which is a striking result. The part of the pressure-transport term that represents the work done by the fluctuating pressure forces is seen to be primarily responsible for the observed shift in the energy balance. The profiles of the energy terms reveal a strong coupling between velocity and pressure fluctuations at large cross-stream distances, and a region of negative production whose magnitude increases with increasing counterflow. These results can help us better understand the physical processes causing absolute instability in a mixing layer. In particular, the streamwise redistribution of disturbance energy by fluctuating pressure, which is "nonlocal" in character for incompressible flows, seems to play a key role in this respect.
\end{abstract}

DOI: 10.1103/PhysRevFluids.6.L091901

\section{INTRODUCTION}

The classification of instabilities in open flows into convective and absolute instability has been an important theme of research in hydrodynamic instability. The convectively unstable flows behave as noise amplifiers, whereas absolutely unstable flows exhibit an intrinsic oscillator behavior [1]. This has implications for technological applications as the method by which a flow can be controlled depends on the nature of instability it undergoes.

Huerre and Rossi [2] have outlined the methods of determining the conditions for the onset of absolute instability for which the dispersion relation is the essential starting point. These techniques have been applied to a variety of flows to determine the type of instability they undergo [3]. In particular, the plane incompressible mixing layer has been studied extensively as a prototypical example of free shear layers undergoing convective-to-absolute transition; see the references in Ref. [3]. Huerre and Monkewitz [1] were the first to determine the conditions for the onset of absolute instability for the plane mixing layer using a simple "tanh" model for the base velocity

\footnotetext{
*Present address: Department of Mathematics, Indian Institute of Technology Madras, Chennai 600036, India.

†Corresponding author: sdiwan@iisc.ac.in
} 
profile [4] given by

$$
\frac{U(y)}{U_{m}}=1+\lambda \tanh \left(\frac{y}{2 \delta_{\omega}}\right),
$$

where $U$ is the streamwise velocity, $y$ is the cross-stream distance, $\delta_{\omega}$ is the vorticity thickness, $U_{m}$ is the average stream velocity $\left[=\left(U_{1}+U_{2}\right) / 2\right], \lambda=\left(U_{1}-U_{2}\right) /\left(U_{1}+U_{2}\right)$, and $U_{1}$ and $U_{2}$ are the velocities of the faster and slower streams, respectively. (An alternative length scale used for nondimensionalization could be the momentum thickness of the mixing layer [1].) Huerre and Monkewitz [1] solved the Rayleigh stability equation, which governs the inviscid instability of the plane parallel flows [2], for the mixing-layer profile in Eq. (1) and found that the absolute instability is triggered when $\lambda$ reaches a critical value of 1.315 . These results were confirmed by the experiments of Strykowski and Niccum [5] on an axisymmetric mixing layer with the mixing-layer thickness much smaller than the jet thickness, thereby serving as a good approximation to the plane mixing layer. They clearly observed the switch in the instability character of the flow from convective to absolute (marked by an emergence of a periodic regime with a well-defined frequency) at a critical value of $\lambda=1.34$, which is close to 1.315 obtained in Ref. [1]; see the discussion in Ref. [2]. The experiments of Ref. [5], thus, supported the validity of the plane parallel approximation and inviscid formulation, inherent in the Rayleigh analysis, for studying the instability of mixing layers. There have been extensions of the incompressible mixing layer to include effects of density stratification, compressibility, viscoelasticity, confinement, etc., for determining their influence on the onset of absolute instability [6-8]. In the present Letter, we limit ourselves to the plane homogeneous incompressible mixing layer within the inviscid parallel instability framework.

It is worth noting that, despite the above studies, the exact mechanism responsible for the onset of absolute instability in a mixing layer (as well as in other shear flows) is not entirely clear. This is partly because most of the previous studies have focused on the behavior of eigenvalues, e.g., the "spatial" branches, as the absolute instability is approached [2]. The corresponding behavior of eigenfunctions in this limit has not been investigated in sufficient detail (except for a few recent studies on separated-flow profiles $[9,10]$ ). In particular, the spatial energy budget for the plane mixing layer approaching absolute instability warrants a close examination. In this Letter, we carry out such an analysis following the treatment of Hama et al. [11] and numerically calculate the different terms in the spatial energy equation for the mixing layer (using linearized disturbance eigenfunctions) to assess their relative importance. We find that the "pressure-transport" term in the spatial energy balance plays an increasingly prominent role in comparison to the "energy production" term as the absolute instability is approached; the former emerges as the primary mechanism of disturbance growth near the threshold of absolute instability for the mixing layer. Interestingly, the part of the pressure-transport term that contributes to the transport of streamwise disturbance energy shows a large variation in this limit. The correlation between velocity and pressure eigenfunctions is found to be mainly responsible in determining the relative significance of the production and pressure-transport terms.

\section{SPATIAL ENERGY BUDGET}

The spatial energy budget for plane parallel flows within the inviscid framework, valid for the convectively unstable flows, is given by ([9])

$$
\begin{aligned}
\frac{d}{d x} \int_{y_{1}}^{y_{2}} U\left(\frac{\overline{u^{\prime 2}}+\overline{v^{\prime 2}}}{2}\right) d y & =\int_{y_{1}}^{y_{2}}\left(-\overline{u^{\prime} v^{\prime}}\right) \frac{d U}{d y} d y+\frac{d}{d x} \int_{y_{1}}^{y_{2}} \frac{\left(-\overline{u^{\prime} p^{\prime}}\right)}{\rho} d y \\
\text { (K.E.T. } & =\text { Prod. + P.T.) }
\end{aligned}
$$

Here $x$ is the streamwise coordinate, $y_{1}$ and $y_{2}$ indicate the lower and upper limits of the domain in the cross-stream direction, $\rho$ is density, and $u^{\prime}, v^{\prime}$, and $p^{\prime}$ indicate fluctuations in the streamwise velocity, cross-stream velocity, and pressure, respectively. The overbar reresents time averaging 
over one time period. The term on the left-hand side in Eq. (2) represents the mean transport of the disturbance kinetic energy in the streamwise direction (to be denoted as "K.E.T."). This essentially represents the spatial growth of the disturbance kinetic energy. The first term on the right-hand side in Eq. (2) is the production of disturbance kinetic energy (to be denoted as "Prod.") and the second term on the right-hand side is the pressure-transport term (denoted as "P.T."), involving the transport of disturbance pressure by the fluctuating streamwise velocity. The pressure-transport term is a distinguishing feature of the spatial energy equation and is not present in the temporal version of the energy equation [11]. The P.T. term can be decomposed into two parts as

$$
\begin{aligned}
\frac{d}{d x} \int_{y_{1}}^{y_{2}} \frac{\left(-\overline{u^{\prime} p^{\prime}}\right)}{\rho} d y & =\int_{y_{1}}^{y_{2}} \overline{\frac{-p^{\prime}}{\rho} \frac{\partial u^{\prime}}{\partial x}} d y+\int_{y_{1}}^{y_{2}} \overline{\frac{-u^{\prime}}{\rho} \frac{\partial p^{\prime}}{\partial x}} d y \\
\text { (P.T. } & =\text { P.T. }- \text { I + P.T. - II). }
\end{aligned}
$$

The first and second terms on the right-hand side in Eq. (3) will be denoted as "P.T.-I" and "P.T. -II," respectively. It can be shown that P.T.-I and P.T.-II contribute to the mean transport of $\left(\overline{v^{\prime 2}} / 2\right)$ and $\left.\overline{\left(u^{\prime 2}\right.} / 2\right)$, respectively [9] as shown in Eq. (4),

$$
\begin{aligned}
& \frac{d}{d x} \int_{y_{1}}^{y_{2}} U\left(\overline{\frac{u^{\prime 2}}{2}}\right) d y=\int_{y_{1}}^{y_{2}}\left(-\overline{u^{\prime} v^{\prime}}\right) \frac{d U}{d y} d y+\int_{y_{1}}^{y_{2}} \overline{\frac{-u^{\prime}}{\rho} \frac{\partial p^{\prime}}{\partial x}} d y, \\
& \frac{d}{d x} \int_{y_{1}}^{y_{2}} U\left(\overline{\frac{v^{\prime 2}}{2}}\right) d y=\int_{y_{1}}^{y_{2}} \overline{\frac{-p^{\prime}}{\rho} \frac{\partial u^{\prime}}{\partial x}} d y .
\end{aligned}
$$

The different terms in Eqs. (2) and (3) have been obtained by numerical integration after substituting the modal representations of fluctuations in the respective terms. The modal representation is given as $\left(u^{\prime}, v^{\prime}, p^{\prime} / \rho\right)=A(\lambda) \frac{1}{2}\left\{[u(y), v(y), p(y)] e^{i(\alpha x-\omega t)}+\right.$ c.c. $\}$, where $\alpha$ is the streamwise wavenumber, $\omega$ is the frequency, $t$ is time, and c.c. indicates the complex conjugate. Here $A(\lambda)$ is an arbitrary complex constant which is function of a given velocity profile [Eq. (1)] and is a consequence of the Rayleigh equation representing an eigenvalue problem. The same arbitrary constant appears for $u^{\prime}, v^{\prime}$, and $p^{\prime}$ as these quantities are derived from the same linearized disturbance streamfunction [12]. In this Letter we use the spatial framework for which $\omega$ is real and specified, and $\alpha=\alpha(\omega)$ is the required eigenvalue. $\alpha=\alpha_{r}+i \alpha_{i}$, where $\alpha_{r}$ represents the streamwise wavenumber and $\alpha_{i}$ represents the spatial growth/decay rate. Our focus here is the growing wave for which $\alpha_{i}<0$. Using the eigenvalue representation, the pressure-velocity correlation term in Eq. (2) can be written as

$$
\frac{\overline{u^{\prime} p^{\prime}}}{\rho}=\frac{|A(\lambda)|^{2}}{4}\left(u p^{*}+p u^{*}\right) e^{-2 \alpha_{i} x}
$$

where $|\cdot|$ represents the amplitude of a complex quantity. The other correlation terms $\left(\overline{u^{\prime 2}}, \overline{v^{\prime 2}}, \overline{u^{\prime} v^{\prime}}\right)$ in Eq. (1) can be expressed in terms of eigenfunctions in a similar fashion. Substituting these in Eq. (2), we get

$$
\left(\frac{-\alpha_{i}}{2}\right) \int_{y_{1}}^{y_{2}} U\left(u u^{*}+v v^{*}\right) d y=\frac{1}{4} \int_{y_{1}}^{y_{2}}\left[-\left(u v^{*}+u^{*} v\right)\right] \frac{d U}{d y} d y+\left(\frac{-\alpha_{i}}{2}\right) \int_{y_{1}}^{y_{2}}\left[-\left(u p^{*}+u^{*} p\right)\right] d y,
$$

where $|A(\lambda)|^{2}$ and $e^{-2 \alpha_{i} x}$ cancel out from both sides. Note that the K.E.T. and P.T. terms are multiplied by the growth rate $\left(-\alpha_{i}\right)$ as they involve streamwise differentiation [Eq. (2)]. Thus, their magnitudes will depend upon the correlation between the respective eigenfunctions as well as the growth rate of the wave. The Prod. term on the other hand is dictated entirely by the eigenfunction correlation.

For the present purposes, the spatial eigenvalues $[\alpha=\alpha(\omega)]$ and eigenfunctions $[u(y), v(y), p(y)]$ for the tanh profiles [Eq. (1)] are obtained by numerically solving the Rayleigh 

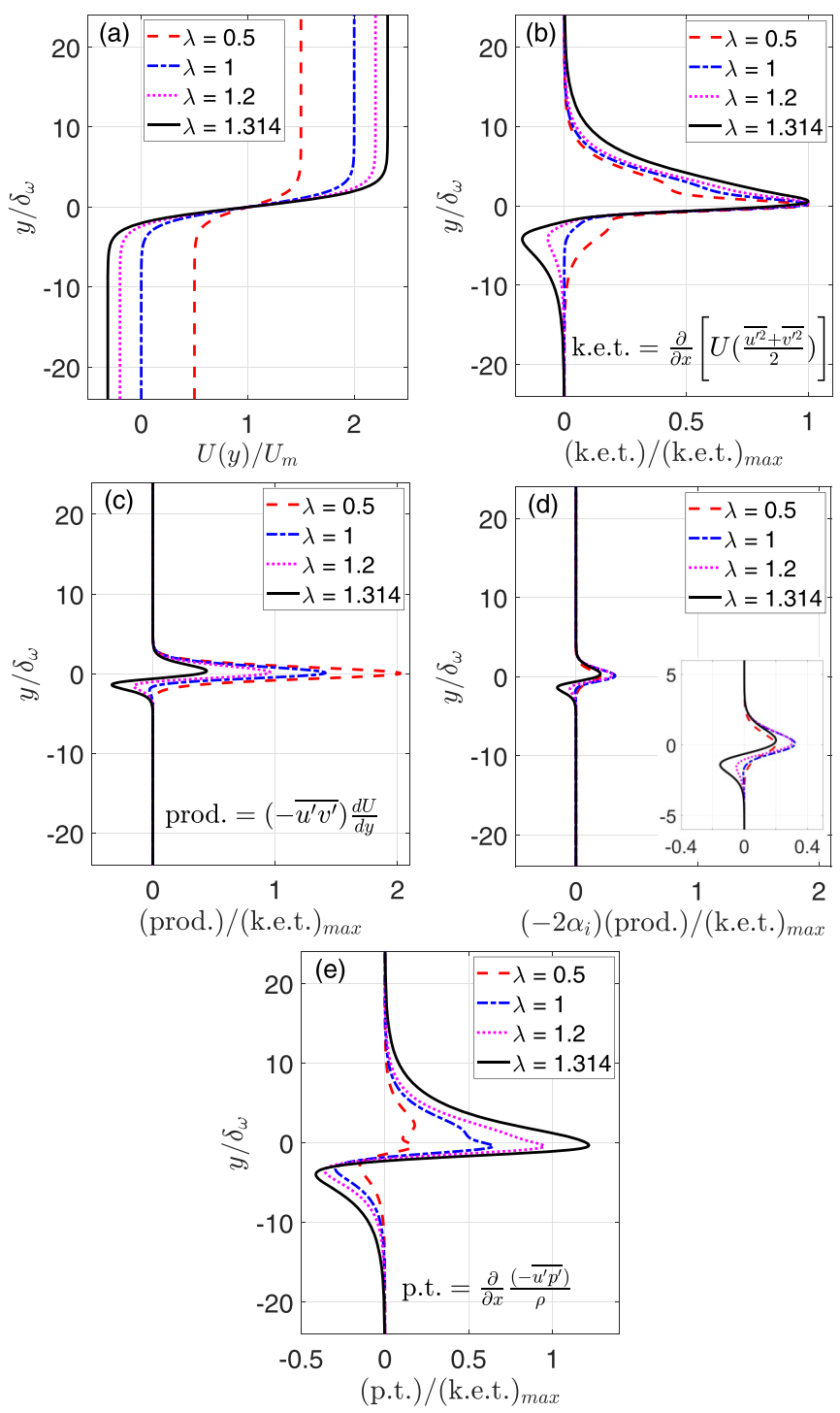

FIG. 1. (a) Base-flow velocity profiles for the tanh mixing layer [Eq. (1)]. (b)-(e) Cross-stream profiles of (b) kinetic-energy transport, (c) energy production, (d) production pre-multiplied with growth rate, and (e) pressure transport. The inset in (d) shows an expanded view of the region near $y=0$. All the quantities in (b)-(e) are normalized by k.e.t. max $_{\text {to }}$ to enable comparison across different values of $\lambda$.

equation using the shooting method; the code has been validated against the known results in the literature. Four different values of $\lambda$ are used for this exercise, namely, $\lambda=0.5,1,1.2$, and 1.314; see Fig. 1(a). A pinch-point analysis is carried out to confirm that these profiles are convectively unstable and that absolute instability occurs at $\lambda=1.315$ [1]. For each $\lambda$ the range of unstable eigenvalues is computed, and the most amplified frequency is determined, denoted as $\omega_{m a}$. The integral terms in the spatial energy equation are calculated for $\omega_{m a}$ for each $\lambda$. Table I lists the most amplified eigenvalues and the contribution of various terms to the energy budget. Note that it is not possible to determine the absolute values of the energy terms because of the presence of the arbitrary constant, and, therefore, we assess the relative contribution of Prod. and P.T. to the energy 
TABLE I. Integral terms in the spatial energy budget for the mixing-layer profiles [Eq. (1)] using Rayleigh analysis. The percentages are expressed in terms of K.E.T.

\begin{tabular}{lcccccccr}
\hline \hline$\lambda$ & $U_{2} / U_{1}$ & $\omega_{m a}$ & $\alpha_{r}\left(\omega_{m a}\right)$ & $\alpha_{i}\left(\omega_{m a}\right)$ & Prod. $(\%)$ & P.T. $(\%)$ & P.T.-I $(\%)$ & P.T. -II (\%) \\
\hline 0.5 & 0.33 & 0.2195 & 0.2193 & -0.0492 & 90.69 & 9.33 & 49.37 & -40.04 \\
1 & 0 & 0.209 & 0.2051 & -0.1142 & 59.06 & 40.97 & 44.56 & -3.59 \\
1.2 & -0.091 & 0.2005 & 0.1866 & -0.1593 & 34.75 & 65.26 & 40.97 & 24.29 \\
1.314 & -0.136 & 0.1925 & 0.1421 & -0.229 & 6.01 & 94.09 & 38 & 56.09 \\
\hline \hline
\end{tabular}

budget by normalizing them with K.E.T.; this also enables comparison across different values of $\lambda$. The normalized Prod. and P.T. terms are listed in Table I as percentages. The balance, K.E.T. $=$ Prod. + P.T., is satisfied to within $0.1 \%$ or better, validating the Rayleigh code.

We observe from Table I that for $\lambda=0.5$ the normalized Prod. term dominates the energy budget and contributes $91 \%$ to the total K.E.T. As $\lambda$ increases from 0.5 to 1.314 , the relative contribution of Prod. reduces significantly, and there is a substantial gain in that of P.T.; both show a monotonic variation with $\lambda$. At $\lambda=1.314$, which is on the verge of becoming absolutely unstable, P.T. dominates the energy balance entirely with Prod. accounting for only $6 \%$ of K.E.T. In other words, at the critical condition of absolute instability for the plane mixing layer, almost entire contribution to K.E.T. comes from P.T., which is a striking result. It is of interest to understand what causes this substantial increase in P.T. (and decrease in Prod.) with an increase in $\lambda$. One possibility is the premultiplying factor $\left(-\alpha_{i}\right)$ appearing in Eq. (6), which increases by a factor of about 4.6 from $\lambda=0.5$ to $\lambda=1.314$ (Table I). However, it appears in both the K.E.T. and P.T. terms and, therefore, does not account for the increase in the contribution of P.T. relative to K.E.T. For the normalized Prod. term, $\left(-\alpha_{i}\right)$ appears in the denominator [Eq. (6)] but still does not account for the considerable decrease in its relative contribution with increase in $\lambda$ (Table I). This suggests that the correlation between the eigenfunctions ( $u, v$ for Prod. and $u, p$ for P.T.) must play a key role in the observed changes in the energy budget as the absolute instability is approached.

Table I also includes the individual contributions of P.T.-I and P.T.-II to the pressure-transport term (as percentages of K.E.T.). P.T.-I shows a weak variation with an increase in $\lambda$, decreasing from about $50 \%$ to $38 \%$ over the range of $\lambda$ considered. On the other hand, P.T.-II shows a significant increase (including a sign change) from $-40 \%$ to $56 \%$ as $\lambda$ increases from 0.5 to 1.314 . Thus, the increasing predominance of P.T. as the absolute instability is approached for the mixing layer is entirely due to P.T.-II (with P.T.-I, in fact, having an opposite effect of decreasing the magnitude of P.T. with increasing $\lambda$ ).

\section{CROSS-STREAM PROFILES OF ENERGY TERMS}

Figures 1(b), 1(c) and 1(e) show the cross-stream distribution of the integrands in the K.E.T., Prod., and P.T. integrals in Eq. (2), i.e., the profiles of kinetic-energy transport (k.e.t.), energy production (prod.), and pressure transport (p.t.). To get rid of the arbitrary constant, the profiles are normalized by the maximum kinetic energy [(k.e.t. $)_{\max }$ ] for each $\lambda$; the same normalization is used for all the profiles (at a given $\lambda$ ) to enable comparison. The peak in the normalized production profiles decreases as $\lambda$ increases from 0.5 to 1.314 , and a region of negative production appears for $\lambda>1$, whose extent increases with increasing $\lambda$ [Fig. 1(c)]. To understand the effect of the multiplying factor $1 /\left(-2 \alpha_{i}\right)$ in the normalized production term [Eq. (6)], we plot the profiles of " $\left(-2 \alpha_{i}\right) *$ prod./(k.e.t. $)_{\max }$ " in Fig. 1(d); this essentially removes the effect of changes in the disturbance growth rate from the normalized production term. We observe an approximate scaling of the region near the production peak, implying that the large decrease in the production peak seen in Fig. 1(c) can be attributed to the $1 /\left(-2 \alpha_{i}\right)$ factor in the normalized production. However, there is also a significant contribution from the negative production region which does not scale 

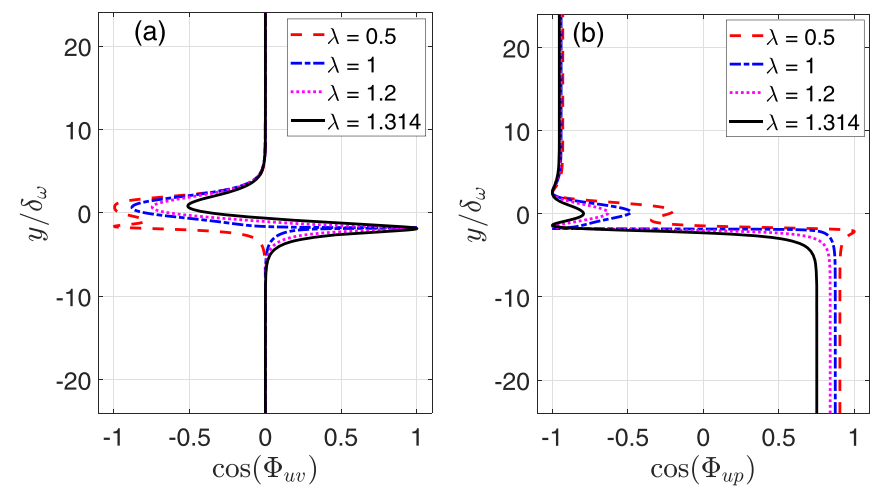

FIG. 2. Cross-stream profiles of (a) $\cos \left(\Phi_{u v}\right)$ and (b) $\cos \left(\Phi_{u p}\right)$ for different values of $\lambda$. $\Phi$ represents phase difference between the two chosen quantities.

with $1 /\left(-2 \alpha_{i}\right)$ and which makes an increasingly negative contribution to the integral production as $\lambda$ approaches 1.314 [Fig. 1(d)]. This explains the significant decrease in the normalized integral production in this limit as seen in Table I. In fact, for $\lambda=1.314$, the negative production nearly equals in magnitude with positive production, consistent with the negligible $(6 \%)$ contribution of this term to the energy budget (Table I). The normalized pressure-transport profiles exhibit regions of positive and negative values for all $\lambda$ 's [Fig. 1(e)]. Interestingly, these profiles show a considerably larger spread in the cross-stream direction in comparison with the production profiles [Figs. 1(c) and 1(e)]. The production profiles are nonzero in the interval $y / \delta_{\omega} \approx \pm 4$ [Fig. 1(c) and 1(d)], which is the region where most of the base-velocity shear is concentrated [Fig. 1(a)]. On the other hand, the pressure-transport profiles reach out to $y / \delta_{\omega} \approx \pm(15-20)$ depending upon $\lambda$ (the higher the reach, the larger the $\lambda$ ), which is four to five times larger than the reach of the production profiles. This shows that pressure fluctuations continue to remain correlated with streamwise velocity fluctuations even in the regions of negligible base-flow shear.

One measure of the correlation (or "coupling") between any two quantities is the phase difference between them; Eq. (7) presents definitions of the phase difference between $u$ and $v$ (i.e., $\Phi_{u v}$ ) and that between $u$ and $p\left(\Phi_{u p}\right)$,

$$
\left(u v^{*}+u^{*} v\right)=2|u||v| \cos \left(\Phi_{u v}\right), \quad\left(u p^{*}+u^{*} p\right)=2|u||p| \cos \left(\Phi_{u p}\right) .
$$

Note that $\cos \left(\Phi_{u v}\right)$ represents the "correlation coefficient" between $u^{\prime}$ and $v^{\prime}$ as commonly defined in the turbulence literature [13]; the same is true for $\cos \left(\Phi_{u p}\right)$. Figures 2(a) and 2(b) show the cross-stream profiles of $\cos \left(\Phi_{u v}\right)$ and $\cos \left(\Phi_{u p}\right)$, respectively, for different values of $\lambda$. First of all, there is considerable evolution in $\Phi_{u v}$ and $\Phi_{u p}$ with an increase in $\lambda$, indicating that the large decrease (increase) in the relative contribution of production (pressure transport) to the energy budget is because of the large changes in the phase relationship between $u$ and $v$ ( $u$ and $p$ ). Note that there is also a contribution from the amplitude profiles $|u(y)|,|v(y)|$, and $|p(y)|$ (not shown here) in determining the energy integrals. Second, for $\left|y / \delta_{\omega}\right|>5, \cos \left(\Phi_{u v}\right)$ is nearly zero whereas $\left|\cos \left(\Phi_{u p}\right)\right|$ is close to 1 [especially for $y / \delta_{\omega}>+4$; Fig. 2(b)]. This shows that there is a strong coupling between velocity and pressure fluctuations away from the region of base-flow shear, supporting our previous observation of a large spread in $y$ of the p.t. profile [Fig. 1(e)]. On the other hand, $u$ and $v$ are uncoupled in this region making the production distribution much more compact [Fig. 1(c)].

The profiles of the two constituent pressure-transport terms in Eq. (3) (p.t.1 and p.t.2) are shown in Figs. 3(a) and 3(b). The distribution of normalized p.t.1 is qualitatively similar to that of p.t. [Fig. 1(e)], with the p.t.1 profiles showing a gradual evolution as $\lambda$ increases from 0.5 to 1.314 [Fig. 3(a)]. The normalized p.t.2 profiles, on the other hand, show a significant evolution with 

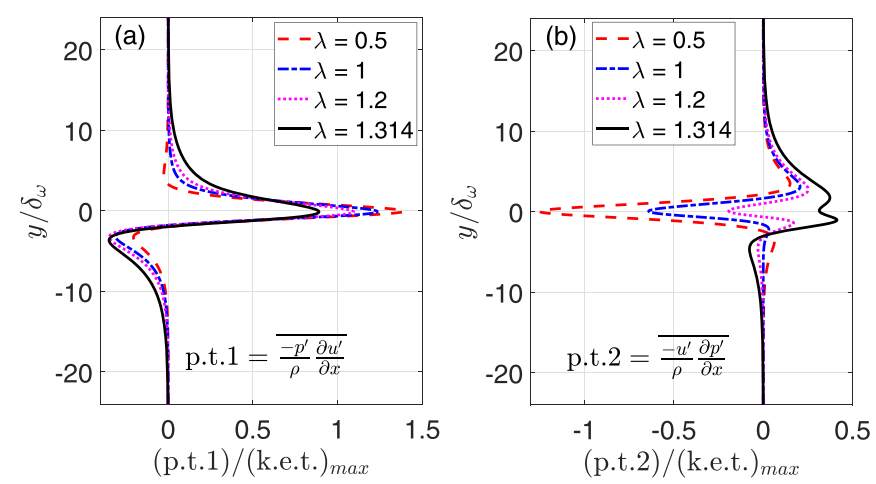

FIG. 3. Cross-stream profiles of the constituent pressure-transport terms: (a) p.t.1 and (b) p.t. 2 for different values of $\lambda$.

increase in $\lambda$ [Fig. 3(b)]. For $\lambda=0.5$, there is a large negative region of p.t. 2 near $y=0$, flanked on either side by a positive lobe of much smaller magnitude. As $\lambda$ increases, this negative region shrinks in size progressively, and the relative contribution of the positive lobes increases [Fig. 3(b)]. At $\lambda=1.314$, the normalized p.t. 2 profile is dominated by positive values with the negative region shrinking to a negligible size. There is also a considerable qualitative difference in the shape of this profile at $\lambda=1.314$ as compared to other $\lambda$ values [Fig. 3(b)]. This behavior is again consistent with the significant increase in the contribution of P.T.-II to the spatial energy balance, along with a sign change as $\lambda$ increases (Table I).

It is worth noting that the terms P.T.-I and P.T.-II (or their corresponding integrands, p.t.1 and p.t.2) have distinct physical interpretations. P.T.-I involves the pressure-strain correlation term, which is known to be responsible for the intercomponent transfer of energy as well as the observed anisotropy in turbulent shear flows [13]. P.T.-II represents the work done by the fluctuating pressure gradients on the streamwise velocity fluctuations. The above results (Table I and Fig. 3) show that the pressure-work term plays an increasingly prominent role as the absolute instability is approached, whereas the contribution of the pressure-strain term does not change much. Equation (4) and Table I show that for $\lambda=0.5$, there is an approximate equipartition of K.E.T. into the transport of streamwise and cross-stream components, respectively, given by Prod. + P.T.-II and P.T.-I. This balance is upset as the mixing layer approaches absolute instability with the transport of streamwise kinetic energy becoming more dominant at $\lambda=1.314$ (Table I).

\section{CONCLUDING REMARKS}

The spatial energy budget for the plane incompressible mixing layer (using tanh profiles) reveals that the integral pressure-transport term becomes increasingly more prominent as compared to the integral production term as the absolute instability is approached. At the threshold of absolute instability, the former completely overwhelms the latter implying that the streamwise pressure transport emerges as a dominant mechanism for disturbance amplification in a mixing layer. The production mechanism (believed to be the primary cause of disturbance growth in unstable flows) is much more subdued near the onset of absolute instability; its contribution to energy budget (normalized on kinetic-energy transport) is $91 \%$ at $\lambda=0.5$, which reduces to mere $6 \%$ at $\lambda=1.314$. The correlation between the pressure and the velocity eigenfunctions is found to dictate the changes in the normalized energy balance with increasing $\lambda$. Notably, a region of negative production appears in the production profiles for $\lambda>1$, whose magnitude increases with $\lambda$, explaining decreasing contribution of the integral production term. The cross-stream distribution of the pressure-transport term is found to be more spread out compared to that of the production term due to a strong coupling between $u^{\prime}$ and $p^{\prime}$ observed at large cross-stream distances. The part of the pressure-transport 
term, viz., $\overline{\frac{-u^{\prime}}{\rho} \frac{\partial p^{\prime}}{\partial x}}$, which represents the work done by the fluctuating pressure forces (and which contributes to the mean transport of streamwise disturbance kinetic energy), is seen to be primarily responsible for the observed shift in the energy balance.

These are interesting results which could help us identify the physical processes that cause the transition from convective to absolute instability for the plane mixing layer. In particular, the dynamics of fluctuating pressure seem to play a key role in this transition. This is conceivable as for incompressible flows pressure is a "nonlocal" quantity [14], and, therefore, it is likely that the velocity fluctuations coupling with those of pressure trigger absolute instability. This aspect has received little attention in the literature and needs further investigation. It is also of interest to extend the present analysis to other flows (both incompressible and compressible) undergoing absolute instability. This exercise is currently underway.

\section{ACKNOWLEDGMENTS}

We thank Professor O. N. Ramesh, Department of Aerospace Engineering, Indian Institute of Science, Bengaluru for suggesting the problem and useful discussions. S.S.D. acknowledges financial support from IISc, Bengaluru in the form of a start-up Grant (Grant No. 1205010620). We thank referees for their useful comments which have been very helpful in improving the quality of the paper.

[1] P. Huerre and P. A. Monkewitz, Absolute and convective instabilities in free shear layers, J. Fluid Mech. 159, 151 (1985).

[2] P. Huerre and M. Rossi, Hydrodynamic instabilities in open flow, in Hydrodynamics and nonlinear instabilities, edited by M. P. Godreche C (Cambridge University Press, Cambridge, UK, 1998).

[3] P. Huerre and P. A. Monkewitz, Local and global instabilities in spatially developing flows, Annu. Rev. Fluid Mech. 22, 473 (1990).

[4] A. Michalke, On spatially growing disturbances in an inviscid shear layer, J. Fluid Mech. 23, 521 (1965).

[5] P. Strykowski and D. Niccum, The stability of countercurrent mixing layers in circular jets, J. Fluid Mech. 227, 309 (1991).

[6] J. Healey, Destabilizing effects of confinement on homogeneous mixing layers, J. Fluid Mech. 623, 241 (2009).

[7] P. Caillol, Absolute and convective instabilities of an inviscid compressible mixing layer: Theory and applications, Phys. Fluids 21, 104101 (2009).

[8] P. K. Ray and T. A. Zaki, Absolute instability in viscoelastic mixing layers, Phys. Fluids 26, 014103 (2014).

[9] S. S. Diwan, Dynamics of early stages of transition in a laminar separation bubble, Ph.D. thesis, Indian Institute of Science, Bangalore, 2009.

[10] M. P. Avanci, D. Rodriguez, and L. S. de B. Alves, A geometrical criterion for absolute instabiity in separated boundary layers, Phys. Fluids 31, 014103 (2019).

[11] F. R. Hama, D. R. Williams, and H. Fasel, Flow field and energy balance according to spatial linear stability theory of the blasius boundary layer, in Laminar-Turbulent Transition, edited by H. F. R. Eppler (Springer-Verlag, Berlin, 1980), pp. 73-85.

[12] P. G. Drazin and W. H. Reid, Hydrodynamic Stability (Cambridge University Press, Cambridge, UK, 1981).

[13] H. Tennekes and J. L. Lumley, A First Course in Turbulence (MIT Press, Cambridge, MA, 1972).

[14] J. Kim, On the structure of pressure fluctuations in simulated turbulent channel flow, J. Fluid Mech. 205, 421 (1989). 